Résumés des conférences et travaux

\title{
Philippe Gauthier (1935-2013)
}

\section{Denis Rousset}

\section{(2) OpenEdition Journals}

Electronic version

URL: https://journals.openedition.org/ashp/1557

DOI: 10.4000/ashp.1557

ISSN: 1969-6310

\section{Publisher}

Publications de l'École Pratique des Hautes Études

\section{Printed version}

Date of publication: 1 September 2014

Number of pages: $x i x-x x i i$

ISSN: 0766-0677

\section{Electronic reference}

Denis Rousset, "Philippe Gauthier (1935-2013)", Annuaire de l'École pratique des hautes études (EPHE) Section des sciences historiques et philologiques [Online], 145 | 2014, Online since 13 November 2014 connection on 06 July 2021. URL: http://journals.openedition.org/ashp/1557 ; DOI: https://doi.org/ 10.4000/ashp. 1557 


\section{PHILIPPE GAUTHIER}

\section{(1935-2013)}

$\mathrm{P}$ philippe Gauthier est décédé le 29 mars dernier. L'ordre du jour de nos assemblées fait qu'hommage lui est rendu alors que nous revenons à la Sorbonne. C'est une coïncidence symbolique pour évoquer la mémoire d'un directeur d'études parmi les plus attachés à l'École pratique à la Sorbonne et à ses locaux patinés par le temps, dont il avait franchi la porte pour la première fois à l'âge de 20 ans.

Né le 15 mai 1935 près de l'Observatoire, rue Cassini, Philippe Gauthier passa à Paris les 22 premières années de sa vie. Son père était un professeur de lettres, également germaniste accompli, qui avait ouvert rue du Cherche-Midi une institution de cours particuliers pour lycéens, où il arriva au lycéen Philippe Gauthier lui-même d'assurer le remplacement d'enseignants absents. C'est dans le Paris du $6^{\mathrm{e}}$ et du $5^{\mathrm{e}}$ arrondissement que Philippe Gauthier grandit, élevé dans la foi catholique, et fit ses études, du collège Stanislas au lycée Louis-le-Grand. Il fut toujours attaché à la laïcité de l'enseignement.

Sans être, disait-il, particulièrement attiré par le professorat, il se tourna vers des études d'histoire à la Sorbonne. Il fit la connaissance d'A. Aymard, qui régnait alors sur l'histoire ancienne, et, en 1955, l'année de son mémoire, il franchit le seuil de notre section, où le même Aymard, directeur d'études à la VI ${ }^{\mathrm{e}}$ section, donnait son enseignement. En 1957, agrégé d'histoire, Philippe Gauthier se vit proposer par A. Aymard un poste d'assistant d'histoire ancienne à l'Institut des hautes études de Tunis, où il partit avec C. Lepelley et où il rencontra J. Desanges. Ainsi, c'est par l'Afrique du Nord qu'il découvrait le pays méditerranéen, les sites de l'ancienne Proconsulaire et l'épigraphie latine. Cette expérience de l'Afrique du Nord se prolongea durablement dans le service militaire dont il accomplit l'essentiel en Algérie, de 1959 à 1962.

De retour d'Algérie, il fut quelques mois professeur au lycée de Saint-Quentin, avant d'être nommé assistant à la Sorbonne. Alors il s'engagea dans une thèse sur les étrangers et la justice dans les cités grecques, et il se tourna, assez tardivement donc, vers l'épigraphie grecque, en commençant à suivre l'enseignement de Louis Robert, à l'École pratique et au Collège de France. À cette époque aussi il se familiarisa avec la numismatique sous la direction de G. Le Rider et fit ses premiers séjours en Grèce. Maître-assistant, puis chargé d'enseignement au collège universitaire de Reims (19661969), il soutint sa thèse en 1970 à l'université de Nancy, et il y devint maître de conférences en 1969, professeur sans chaire en 1972, professeur titulaire en 1974. Très proche de ses collègues É. Will, P. Charneux, P. Goukowsky, C. Brixhe et le doyen J. Schneider, profitant là de conditions de travail qu'il disait n'avoir guère retrouvées plus tard, il s'enracina profondément en Lorraine, avec son épouse Geneviève et leurs quatre enfants.

Aussi ne fut-ce pas sans vaincre quelque hésitation qu'il se résolut à être candidat à la IVe section à la succession de L. Robert, sur la demande de celui-ci. Car sa modestie le faisait également douter qu'il fût à la hauteur de la tâche proposée. Élu à la direction d'études intitulée « Épigraphie et institutions grecques » en 1974 comme directeur 
cumulant, il devint non-cumulant en 1978. Fidèle à notre établissement jusqu'à sa retraite en 2002, il déclina bien des propositions : ainsi de se présenter à un poste de professeur dans une université parisienne, de siéger au jury de l'agrégation alors même que la question au programme était directement inspirée par un de ses livres, de séjourner dans telle institution prestigieuse outre-Atlantique, sans parler de nombreuses réunions internationales où il se refusa à aller.

C'est que Philippe Gauthier entendait continuer à se consacrer à sa famille, à son enseignement et à son œuvre écrite. Travailleur acharné, souhaitant n'être dérangé chez lui par le téléphone qu'à des heures très précises, il nous laisse une œuvre écrite comptant quelque 220 titres. Mais toujours il garda vis-à-vis de cette œuvre une distance mêlée d'ironie, au point que, lorsqu'on lui dit que la bibliographie de Philippe Gauthier constituée à partir de L'Année philologique comptait autant de titres, il répondit : «ce doit être un homonyme... $»^{1}$.

Parmi les cinq ouvrages dont il est seul auteur, ses deux maîtres livres, Symbola. Les étrangers et la justice dans les cités grecques, paru en 1972, et Les cités grecques et leurs bienfaiteurs (IV $V^{e} I^{\text {er }}$ s. avant J.-C.). Contribution à l'histoire des institutions, paru en 1985, indiquent les deux axes majeurs de son œuvre. D'une part l'histoire des institutions grecques, qu'il avait fait figurer dans le titre de sa direction d'études et qu'il remettait ainsi au premier plan en France, renouant aux Hautes études avec l'enseignement il y a un siècle de B. Haussoullier. Grand lecteur d'Aristote, rompu au droit grec ancien et aux subtilités de ses commentateurs, notamment germanophones, P. Gauthier s'est imposé en France et internationalement comme le spécialiste des institutions politiques grecques. Ayant débuté ses enquêtes par la situation juridique des étrangers et par les procédures judiciaires, il élargit ses investigations à l'ensemble des institutions qui font le citoyen en Grèce et définissent sa participation aux cadres politiques, juridiques et fiscaux. Éphébie, institutions militaires, gymnase, modes d'acquisition de la citoyenneté, assemblées, procédures de vote, magistratures, fiscalité, recours aux juges étrangers, traités entre cités : tels sont quelques-uns des thèmes à travers lesquels il traita toutes les facettes de l'« animal politique » qu'était l'homme grec.

L'autre axe majeur est l'histoire politique et sociale et le statut des cités grecques dans une période qu'il a contribué à définir, dans le sillage de L. Robert, en décalage par rapport à la périodisation traditionnelle de l'histoire grecque. C'est non pas la période classique, $\mathrm{V}^{\mathrm{e}}$ et $\mathrm{IV}^{\mathrm{e}} \mathrm{s}$., ni toute la période hellénistique, du $\mathrm{III}^{\mathrm{e}}$ au $\mathrm{I}^{\mathrm{er}} \mathrm{S}$., mais la période allant d'environ 400 à 100 avant notre ère, que par prédilection il étudia, comme l'apogée des cités grecques en tant que corps de citoyens organisés en démocraties. D'une part il contribua à définir les ruptures et les continuités institutionnelles et sociologiques avec les périodes antérieure et postérieure ; d'autre part il traça la césure qui traversait ces siècles, opposant haute époque hellénistique et basse époque hellénistique ; enfin il définit le fonctionnement de ces sociétés par rapport à leurs bienfaiteurs, qu'ils fussent des citoyens, des étrangers, des rois ou, plus tard, des Romains, pointant ainsi sa divergence par rapport au système de gouvernement par l'évergétisme qu'avait mis en avant P. Veyne.

1. Bibliographie de P. Gauthier dans Études d'histoire et d'institutions grecques. Choix d'écrits, VIII756 p., Genève, 2011 (Hautes études du monde gréco-romain, 47), p. 675-698. 
Sans être un intellectuel engagé, Philippe Gauthier était passionné par la politique et par l'histoire contemporaines. Profondément démocrate et européen convaincu, il se disait «plutôt conservateur de tempérament»: il se sentait « de droite avec les hommes de gauche et de gauche avec les hommes de droite ». Philippe Gauthier fut également influencé par la personnalité et l'œuvre de R. Aron, qui l'amenèrent à collaborer à la revue Commentaire. S'il se gardait des généralisations sociologiques et dénonça l'application mécanique en histoire ancienne des idéaux-types inspirés de Max Weber, son vif intérêt pour la philosophie et l'économie politiques l'amena à commenter un des traités de Xénophon, en un livre, Un commentaire historique des Poroi de Xénophon, paru en 1976 dans nos collections, et la philosophie politique d'Aristote, en plusieurs articles et en une traduction commentée de la Constitution des Athéniens, qu'il a depuis plus de trois décennies rédigée : cette traduction commentée paraître en 2014 dans le volume I de l'Aristote de la collection de la Pléiade.

Son quatrième livre, publié aussi par l'EPHE, Nouvelles inscriptions de Sardes (1988), marqua pour ainsi dire une inflexion, liée à l'héritage de Louis Robert. De ce dernier il avait en effet recueilli la charge de publier un lot d'importantes inscriptions trouvées dans la capitale de la Lydie : d'historien qu'il était, il devint alors également épigraphiste de terrain. Il conçut un vif goût pour le travail sur les pierres, qu'il allait poursuivre sur d'autres sites d'Asie Mineure, à Xanthos et à Claros, où il fut appelé dans les années 1990.

C'est aussi l'héritage de Louis Robert qui lui valut de prendre, volens nolens, la direction du Bulletin épigraphique, que B. Haussoullier avait fondé en 1888 dans la Revue des études grecques. En 1987, P. Gauthier reprit les rênes de ce recensement critique, pour diriger une douzaine de collaborateur unis, écrivait-il, par « l'estime réciproque et l'amitié ». Ce ne sont pas moins de 18 livraisons, totalisant plus de 2600 pages, qu'il publia entre 1987 et 2005, en assumant la direction et en partie la rédaction. C'est peu dire que chacun y attendait son jugement, tant sur les éditions de textes nouveaux que sur les dernières exégèses historiques s'appuyant sur l'épigraphie.

L'autorité de ses avis fit de lui un juge révéré, voire redouté dans les jurys et dans les expertises, que lui-même ne recherchait pas. Car Philippe Gauthier portait sur la corporation un regard volontiers humoristique. Cela ne l'empêcha de recevoir plus d'une marque de reconnaissance à l'étranger et en France, telle son élection à l'Académie des inscriptions et belles-lettres en 2001. Parisien par sa jeunesse et ses études, il était tout sauf d'esprit parisien. Dédaignant, lui l'historien des institutions, les vanités institutionnelles, il se sentait à l'aise un peu en marge : en témoignent les domiciles qu'il élut lorsque la carrière le ramena à la capitale, Poissy tout d'abord, où il habita, à la faveur de relations familiales, la maison du peintre Ernest Meissonnier, puis Évreux, qu'il choisit pour sa retraite.

Mais gardons-nous de penser que P. Gauthier ait été un savant solitaire. Car, dans son métier, c'est à l'enseignement qu'il attachait la plus grande importance. « J'aurais souhaité être instituteur d'école maternelle », disait-il, inspiré aussi qu'il était par l'attachement à sa famille, ses enfants et ses treize petits-enfants. Exceptée la catéchèse, c'est devant des élèves plus âgés que l'a conduit sa carrière. De son enseignement durant 28 années à l'École pratique, il a mis son point d'honneur à donner le rapport annuel pour le présent Annuaire et il a évoqué les grandes satisfactions qu'il 
avait reçues. Les élèves qu'il y avait eus, il n'hésitait pas à les appeler, aux côtés de ses professeurs A. Aymard et L. Robert, son « troisième maître ». Soulignant ainsi les connaissances qu'il avait ainsi acquises lui-même, il passait sous silence les progrès bien plus considérables que fit faire à tant d'auditeurs son enseignement exigeant, et toujours empreint d'humour, voire de causticité. À l'École pratique, où dans des débats passionnés il montra sa pondération et où il participa à l'administration de l'école doctorale, il se disait « indéfectiblement attaché » : "Cet admirable établissement que les étrangers nous envient, mais que certains bureaucrates sont périodiquement tentés de réformer "en maniant le rabot pour tout unifier au niveau de la planche" selon la formule de Louis Robert », écrivait en 2002 P. Gauthier.

Bien que, tout comme Louis Robert, il pourfendît l'obsession du travail en équipe, il accepta, sur l'invitation de Jean-Louis Ferrary, de diriger un programme de recherches sur les institutions hellénistiques. Ce programme, qui lui permettait pour ainsi dire de prolonger son magistère au-delà du mardi matin, déboucha sur un colloque, en quelque sorte un hommage à son œuvre, paru dans nos collections en 2005, comme aussi enfin son Choix d'écrits, paru en 2011.

C'est cependant avant tout la discussion en tête-à-tête qu'il préférait, suivant la maxime de son ami Édouard Will : « on ne discute bien qu'à deux ». Il mettait en pratique cette maxime, lisant avec la plus grande attention les travaux avant de recevant longuement leurs auteurs, et de les diriger en homme de savoir, jamais de pouvoir.

La discrétion personnelle, voire la pudeur qui étaient les siennes recommanderaient que l'on évoque ici seulement ses qualités de collègue et de savant, et non sa personne même. Mais sa personnalité est certainement ce qui a le plus marqué ceux que le métier a rapprochés de lui. Catholique convaincu et pratiquant, décédé le vendredi de Pâques, il ne cessa de suivre avec compréhension et sollicitude ceux qu'il avait formés, toujours attentif aux autres exigences qui pesaient sur eux, celles de l'enseignement et de la vraie vie. Dans ces domaines aussi, il fut un tuteur sans pareil : son autorité bienveillante, l'acuité de ses avis, tout autant que de son regard, sa fine sensibilité ont fait de lui pour ses élèves un véritable recteur de leurs études, et bien au-delà. 